УДК 517.9

\title{
Fictitious Domain Method for Equilibrium Problems of the Kirchhoff-Love Plates with Nonpenetration Conditions for Known Configurations of Plate Edges
}

\author{
Nyurgun P. Lazarev* \\ Vladimir V. Everstov ${ }^{\dagger}$ \\ Natalya A. Romanova ${ }^{\ddagger}$ \\ Institute of Mathematics and Information Science \\ North-Eastern Federal University \\ Belinsky, 58, Yakutsk, 677000 \\ Russia
}

Received 29.07.2019, received in revised form 10.08.2019, accepted 20.09.2019

\begin{abstract}
New models are investigated in this paper, that describe equilibrium states of plates with Signorini type nonpenetration conditions. In these models, it is assumed that under appropriate loading, plates have special deformations with already known configurations of edges. For this case, we deal with new nonpenetration conditions that allow us to describe more precisely the possibility of contact interaction of plate edges. Using the method of fictitious domains, it is proved that an original contact problem for a plate can be obtained by passing to the limit when a rigidity parameter tends to infinity from a family of auxiliary problems formulated in a wider domain. The mentioned family of problems model an equilibrium state of plates with a crack and depend on the positive rigidity parameter. For these problems, to prevent a mutual penetration of the opposite crack faces boundary conditions of inequality type are imposed on the inner boundary corresponding to the crack. For the problem, describing a plate with a crack that intersects the external boundary at zero angle (a case of a boundary having one cusp), the unique solvability is proved.
\end{abstract}

Keywords: Signorini condition, fictitious domain, non-penetration condition, Kirchhoff-Love plate, crack. DOI: 10.17516/1997-1397-2019-12-6-674-686.

\section{Introduction}

The fictitious domain method for nonlinear models, describing equilibrium of $n$-dimensional ( $n=2,3$ ) elastic bodies with nonlinear boundary conditions in the form of inequalities was elaborated in [1-10]. Particularly, non-linear models for plates are studied in [6-9]. Furthermore, as was proved in [1], the two-dimensional contact problem for a rigid body with a Signorini type boundary condition can be considered as a limiting case for a family of equilibrium problems modelling two-dimensional bodies with cracks. Analogous results for the Kirchhoff-Love plates were established in $[6,9]$.

\footnotetext{
*nyurgun@ngs.ru

†vv.everstov@s-vfu.ru

${ }^{\ddagger}$ nan.romanova@s-vfu.ru

(C) Siberian Federal University. All rights reserved
} 
The method of the fictitious domain has proven useful in establishing the solvability of problems that describe equilibrium of bodies with cracks crossing the external boundary at zero angles $[2,4,8,9]$. In the last years, within the framework of crack models subject to non-penetration boundary conditions, numerous works have been published, see, for example, [11-25].

We should note that all boundary value problems considered here are related to the class of free boundary problems with inequality type boundary conditions. About fictitious domain method for linear problems, one can find in [26].

In the present work, models describing the equilibrium of plates under the Kirchhoff-Love hypothesis are investigated. We have considered two models for plates, for the first one, we study a plate that may be subjected to mechanical contact with a rigid obstacle, and the second corresponds to a plate with a crack. Boundary conditions are given as a system of two inequalities and describe non-penetration for a case of previously known configurations near plate edges that can be subjected to mechanical contact. For the original problem, we assume that in a strainfree state, a part of the cylindrical surface corresponding to the plate contour is adjacent to the rigid obstacle. A unilateral condition of Signorini's type is imposed on a given part $\gamma$ of the boundary $\Gamma_{1}$ of a domain $\Omega_{1}$ that determines the plate's midsurface. This condition characterizes a possibility of a certain mechanical contact interaction between the plate and the obstacle. On the rest part $\Gamma_{0}$ of the boundary $\Gamma_{1}$, meas $\Gamma_{0}>0, \Gamma_{0} \cap \gamma=\emptyset, \Gamma_{0} \cup \gamma=\Gamma_{1}$, we consider a clamping condition. Auxiliary problems are formulated in extended domains $\Omega_{\gamma}=\Omega \backslash \bar{\gamma}\left(\Omega_{1} \subset \Omega_{\gamma}\right)$ that describe the equilibrium of plates with a crack. For these problems, a condition of mutual nonpenetration of opposite crack faces is given as a system of two inequalities on the crack curve $\gamma$. It is proved that the original contact problem can be obtained from a family of the auxiliary problems by using a passage to the limit when a rigidity parameter tends to infinity. Applying the fictitious domain method, the solvability of an equilibrium problem for a plate with a crack crossing the external boundary at zero angle (in this case, the boundary of a domain does not satisfy the Lipschitz condition) is proved.

\section{Formulation of a contact problem}

Let $\Omega_{1} \subset R^{2}$ be a bounded connected domain with smooth boundary $\Gamma_{1}=\gamma \cup \Gamma_{0}, \gamma \cap \Gamma_{0}=\emptyset$, meas $\Gamma_{0}>0$ (Fig.1). We suppose that the curve $\gamma$ does not contain endpoints. We denote by $\nu=\left(\nu_{1}, \nu_{2}\right)$ the outward unit normal vector to $\Gamma_{1}$. We assume that the plate has a constant thickness $2 h=2$. We introduce the Cartesian coordinates $\left\{x_{1}, x_{2}, z\right\}$ in such a way that the set $\Omega_{1} \times\{0\} \subset \mathbb{R}^{3}$ corresponds to the initial undeformed plane midsurface of the plate.

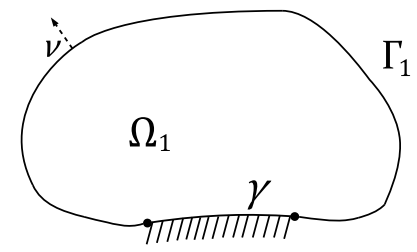

Fig. 1. Geometrical objects of the problem

We denote by $\chi=(W, w)$ the vector of displacements of points of the midsurface, where $W=W(x)=\left(w_{1}, w_{2}\right)$ and $w=w(x)$ are horizontal (along the plane $\left(x_{1}, x_{2}\right)$ ) and vertical displacements respectively, $x=\left(x_{1}, x_{2}\right)$. In accordance with the Kirchhoff-Love hypothesis, for 
displacements of plate points we have the following linear relationships [27]

$$
W^{z}(x, z)=W(x)-z \nabla w, \quad w^{z}(x, z)=w(x), \quad|z| \leqslant 1,
$$

where $|z|$ is a distance from the mid-surface. The strain and integrated stress tensors are denoted by $\varepsilon_{i j}=\varepsilon_{i j}(W), \sigma_{i j}=\sigma_{i j}(W)$, respectively [11]:

$$
\varepsilon_{i j}(W)=\frac{1}{2}\left(w_{j, i}+w_{i, j}\right), \quad \sigma_{i j}(W)=a_{i j k l} \varepsilon_{k l}(W), \quad i, j=1,2,
$$

where $\left\{a_{i j k l}\right\}$ is the given elasticity tensor, assumed to be symmetric and positive definite:

$$
\begin{array}{r}
a_{i j k l}=a_{k l i j}=a_{j i k l}, \quad i, j, k, l=1,2, \quad a_{i j k l} \in L^{\infty}\left(\mathbb{R}^{2}\right), \\
a_{i j k l} \xi_{i j} \xi_{k l} \geqslant c_{0}|\xi|^{2}, \quad \forall \xi, \quad \xi_{i j}=\xi_{j i}, \quad i, j=1,2, \quad c_{0}=\text { const }>0 .
\end{array}
$$

Hereinafter, we will use a summation convention over repeated indices and the following notation

$$
\phi,_{i}=\frac{\partial \phi}{\partial x_{i}}, \quad \phi,_{i j}=\frac{\partial^{2} \phi}{\partial x_{i} \partial x_{j}} .
$$

Next we denote the bending moments by formulae [11]

$$
m_{i j}(w)=-d_{i j k l} w, k l, \quad i, j=1,2,
$$

where the tensor $\left\{d_{i j k l}\right\}$ has the same properties as the tensor $\left\{a_{i j k l}\right\}$. Let $B(S, \cdot, \cdot)$ be a bilinear form defined by the equality

$$
B(S, \chi, \bar{\chi})=\int_{S}\left\{\sigma_{i j}(W) \varepsilon_{i j}(\bar{W})-m_{i j}(w) \bar{w}, i j\right\} d x,
$$

where $S$ is some domain, $\chi=(W, w) \in H^{1}(S)^{2} \times H^{2}(S), \bar{\chi}=(\bar{W}, \bar{w}) \in H^{1}(S)^{2} \times H^{2}(S)$. The potential energy functional of the plate has the following representation [11]:

$$
\Pi\left(\Omega_{1}, \chi\right)=\frac{1}{2} B\left(\Omega_{1}, \chi, \chi\right)-\int_{\Omega_{1}} F \chi d x, \quad \chi=(W, w),
$$

where the vector $F=\left(f_{1}, f_{2}, f_{3}\right) \in L_{\text {loc }}^{2}\left(\mathbb{R}^{2}\right)^{3}$ describes the body forces [11].

Suppose that the following boundary clamping conditions are satisfied

$$
w=\frac{\partial w}{\partial \nu}=W=0 \text { on } \Gamma_{0},
$$

where $\nu$ is the unit external normal vector to $\Gamma_{1}$. We will need the following spaces

$$
\begin{gathered}
H^{1,0}\left(\Omega_{1}\right)=\left\{v \in H^{1}\left(\Omega_{1}\right) \mid v=0 \text { on } \Gamma_{0}\right\}, \\
H^{2,0}\left(\Omega_{1}\right)=\left\{v \in H^{2}\left(\Omega_{1}\right) \mid v=\frac{\partial v}{\partial \nu}=0 \text { on } \Gamma_{0}\right\}, \\
H\left(\Omega_{1}\right)=H^{1,0}\left(\Omega_{1}\right)^{2} \times H^{2,0}\left(\Omega_{1}\right) .
\end{gathered}
$$

Let us write out a boundary condition of Signorini's type for the edge of the plate describing a possible mechanical frictionless contact with a rigid obstacle for the case of a known certain configuration of a plate bending near the edge

$$
W \nu-\frac{\partial w}{\partial \nu} \leqslant 0, \quad \frac{\partial w}{\partial \nu} \leqslant 0 \quad \text { on } \quad \gamma .
$$




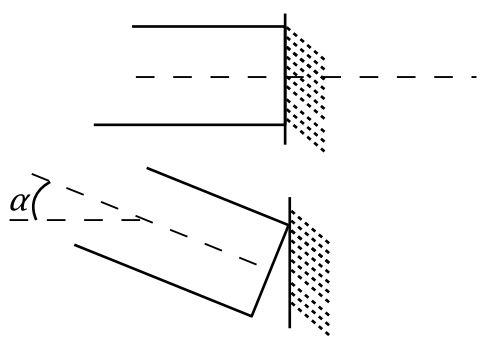

Fig. 2. The configuration of the plate bending near the contact surface of a rigid obstacle

As an example of the considered configuration of the plate edge near the contact surface, which is given by the sign of the expression $\frac{\partial w}{\partial \nu}$, we can provide the following figure for the section of the plate (see Fig. 2), where the angle $\alpha=\arctan \left(\frac{\partial w}{\partial \nu}\right) \leqslant 0$ determines a bending angle.

Here, the top figure shows the state of the plate before deformation, and the bottom one depicts the deformed state. It is easy to see, that if the function $\chi=(W, w)$ satisfies $(4)$, then the following relation

$$
\left(W^{z}(x, z), w^{z}(x, z)\right) \cdot(\nu, 0) \leqslant 0 \quad \text { on } \quad \gamma \quad \forall z:|z| \leqslant 1
$$

also holds. This means that (4) provides non-penetration for a certain known configuration of a plate bending near the edge. The general non-penetration condition has the form [6]

$$
W \nu+\left|\frac{\partial w}{\partial \nu}\right| \leqslant 0 \quad \text { on } \quad \gamma
$$

Now we can specify the following set of admissible displacements

$$
K_{s}=\left\{\chi=(W, w) \in H\left(\Omega_{1}\right) \mid \chi \quad \text { satisfies }(4)\right\} \text {. }
$$

It is obvious that the set $K_{s}$ is convex and closed and, consequently, weakly closed in the reflexive space $H\left(\Omega_{1}\right)$. Applying the Korn and the Poincare inequalities, we infer the following estimates

$$
c_{1}\|W\|_{H^{1,0}\left(\Omega_{1}\right)}^{2} \leqslant \int_{\Omega_{1}} \sigma_{i j}(W) \varepsilon_{i j}(W) d x, \quad c_{2}\|w\|_{H^{2,0}\left(\Omega_{1}\right)}^{2} \leqslant-\int_{\Omega_{1}} m_{i j}(w) w,_{i j} d x,
$$

with constants $c_{1}>0, c_{2}>0$ being independent of $W \in H^{1,0}\left(\Omega_{1}\right)^{2}, w \in H^{2,0}\left(\Omega_{1}\right)$. From this we get the inequality

$$
\|\chi\|_{H\left(\Omega_{1}\right)}^{2} \leqslant B\left(\Omega_{1}, \chi, \chi\right) \quad \forall \chi \in H\left(\Omega_{1}\right),
$$

which guarantees the equivalence of the standard $H\left(\Omega_{1}\right)$ norm with that defined by the seminorm $B\left(\Omega_{1}, \cdot \cdot \cdot\right)$.

A variational statement of an equilibrium problem for an elastic plate that may be subjected to mechanical contact with a rigid obstacle is formulated as follows:

$$
\text { Find } \xi_{s} \in K_{s} \quad \text { such that } \Pi\left(\Omega_{1} ; \xi_{s}\right)=\inf _{\eta \in K_{s}} \Pi\left(\Omega_{1} ; \eta\right)
$$

The estimate (7) and the linearity of the functional $F^{*}: H\left(\Omega_{1}\right) \rightarrow \mathbb{R}, F^{*}(\chi)=\int_{\Omega_{1}} F \chi d x$, guarantee that the energy functional $\Pi\left(\Omega_{1}, \chi\right)$ is coercive and weakly lower semicontinuous on $H\left(\Omega_{1}\right)$ (see [4]). In virtue of the mentioned properties of the functional $\Pi\left(\Omega_{1}, \chi\right)$ and the set $K_{s}$ of 
admissible displacements, we can apply the Weierstrass theorem, which gives an existence of a solution to the problem (8). Uniqueness of the solution $\xi_{s}=\left(U_{s}, u_{s}\right)$ follows from the strict monotonicity of the derivative operator $\Pi_{\eta}^{\prime}\left(\Omega_{1}, \chi\right): H\left(\Omega_{1}\right) \rightarrow H\left(\Omega_{1}\right)^{*}$. Because of the convexity and differentiability of the functional $\Pi\left(\Omega_{1}, \chi\right)$ on $H\left(\Omega_{1}\right)$, the minimization problem $(8)$ is equivalent to the following variational inequality, see [4]

$$
\xi_{s} \in K_{s}, \quad B_{1}\left(\Omega_{1}, \xi_{s}, \chi-\xi_{s}\right) \geqslant \int_{\Omega_{1}} F\left(\chi-\xi_{s}\right) d x \quad \forall \chi \in K_{s} .
$$

\section{Auxiliary problems}

As it turned out, the problem (9) which is equivalent to (8) can be obtained as a limit problem for a family of auxiliary problems formulated in a wider domain than $\Omega_{1}$. Moreover, each problem of this family governs the equilibrium state of an elastic plate with a crack. We extend the domain $\Omega_{1}$ to a domain $\Omega_{\gamma}$ by adding some fictitious domain $\Omega_{2}$ in such a way that $\gamma$ lies strictly inside $\Omega=\operatorname{int}\left(\overline{\Omega_{1} \cup \Omega_{2}}\right)$ (see Fig. 3). We assume that the boundary $\Gamma_{2}$ of $\Omega_{2}$ is sufficiently smooth. The extended domain with cut is denoted by $\Omega_{\gamma}=\Omega \backslash \bar{\gamma}$, and its exterior boundary is denoted by $\Gamma=\left(\Gamma_{1} \cup \Gamma_{2}\right) \backslash \Sigma$, where $\Sigma=\operatorname{int}\left(\Gamma_{1} \cap \Gamma_{2}\right)$. We assume that meas $\left(\Gamma \cap \Gamma_{i}\right)>0, i=1,2$. In accordance with the direction $\nu$, we will use the notations $\Sigma^{+}, \Sigma^{-}$for positive and negative faces of the curve $\Sigma$, respectively. Thus, one can define the traces $v^{+}=\left.v\right|_{\Sigma^{+}}, v^{-}=\left.v\right|_{\Sigma^{-}}$for an arbitrary $v \in H^{1}\left(\Omega_{\gamma}\right)$. The jump of $v$ on $\Sigma$ is denoted by $[v]=v^{+}-v^{-}$. Similar notation will be used for traces and jumps on $\gamma$.

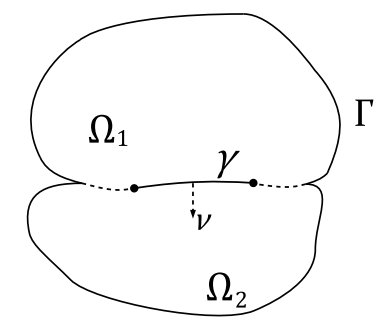

Fig. 3. Geometry of the extended domain

Let us define a family of variational problems that are formulated in $\Omega_{\gamma}$ and depend on the positive parameter $\lambda$, which will subsequently tend to zero. We consider the plates with a midsurface corresponding to the set $\left\{\Omega_{\gamma}\right\} \times 0$. Suppose the elastic coefficients in the subdomain $\Omega_{1}$ are constant, and in the subdomain $\Omega_{2}$ depend on $\lambda$ as follows

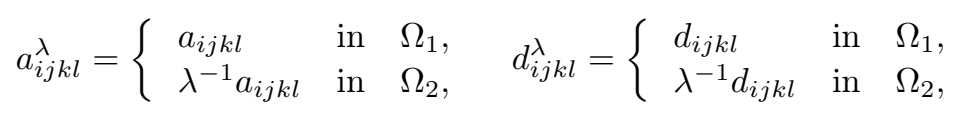

We will need the following spaces

$$
\begin{gathered}
H^{1,0}\left(\Omega_{\gamma}\right)=\left\{v \in H^{1}\left(\Omega_{\gamma}\right) \mid v=0 \text { on } \Gamma\right\} \\
H^{2,0}\left(\Omega_{\gamma}\right)=\left\{v \in H^{2}\left(\Omega_{\gamma}\right) \mid v=\frac{\partial v}{\partial n}=0 \text { on } \Gamma\right\}, \\
H\left(\Omega_{\gamma}\right)=H^{1,0}\left(\Omega_{\gamma}\right)^{2} \times H^{2,0}\left(\Omega_{\gamma}\right),
\end{gathered}
$$


where $n$ is a unit exterior vector to $\Gamma$. For the functions $\chi=(W, w) \in H\left(\Omega_{\gamma}\right)$, $\bar{\chi}=(\bar{W}, \bar{w}) \in H\left(\Omega_{\gamma}\right)$ we can define the following bilinear form

$$
B^{\lambda}\left(\Omega_{\gamma}, \chi, \bar{\chi}\right)=\int_{\Omega_{\gamma}}\left\{\sigma_{i j}^{\lambda}(W) \varepsilon_{i j}(\bar{W})-m_{i j}^{\lambda}(w) \bar{w}, i j\right\} d x
$$

where

$$
m_{i j}^{\lambda}(w)=-d_{i j k l}^{\lambda} w, k l, \quad \sigma_{i j}^{\lambda}(W)=c_{i j k l}^{\lambda} \varepsilon_{k l}(W), \quad i=1,2 .
$$

Obviously, taking into account the formulas (1), (2) for $\sigma_{i j}(W), m_{i j}(w), i, j=1,2$, we can rewrite $B^{\lambda}\left(\Omega_{\gamma}, \cdot, \cdot\right)$ in the form

$$
B^{\lambda}\left(\Omega_{\gamma}, \chi, \bar{\chi}\right)=B\left(\Omega_{1}, \chi, \bar{\chi}\right)+\lambda^{-1} B\left(\Omega_{2}, \chi, \bar{\chi}\right) .
$$

Let us assume that the energy functional of the plate with the crack is defined by

$$
\Pi_{\lambda}\left(\Omega_{\gamma} ; \chi\right)=\frac{1}{2} B^{\lambda}\left(\Omega_{\gamma}, \chi, \chi\right)-\int_{\Omega_{\gamma}} F \chi d x, \quad \chi=(W, w),
$$

where the function $F$ of external forces was given in (3).

Under assumption that for equilibrium state the plate edges have a certain configuration, the non-penetration condition for the crack faces takes the form

$$
[W \nu] \geqslant\left[\frac{\partial w}{\partial \nu}\right], \quad\left[\frac{\partial w}{\partial \nu}\right] \geqslant 0 \quad \text { on } \gamma .
$$

The corresponding equilibrium problem implies the minimization of the energy functional:

$$
\text { find } \xi^{\lambda} \in K \quad \text { such that } \Pi_{\lambda}\left(\Omega_{\gamma} ; \xi^{\lambda}\right)=\inf _{\chi \in K} \Pi_{\lambda}\left(\Omega_{\gamma} ; \chi\right),
$$

where

$$
K=\left\{\chi \in H\left(\Omega_{\gamma}\right) \mid \chi=(W, w) \text { satisfies } \quad(14)\right\}
$$

set of admissible displacements.

Considering that the desired function belongs to the class $H\left(\Omega_{\gamma}\right)$, we have the following relations

$$
[\chi]=0, \quad\left[\frac{\partial w}{\partial \nu}\right]=0 \quad \text { on } \quad \Sigma \backslash \gamma,
$$

i.e. the displacement vector $\chi=(W, w)$ satisfies the gluing conditions on $\Sigma \backslash \gamma$. For the domain $\Omega_{2}$, we introduce the Sobolev spaces

$$
H\left(\Omega_{2}\right)=\left\{\eta=(W, w) \in H^{1}\left(\Omega_{2}\right) \times H^{1}\left(\Omega_{2}\right) \times H^{2}\left(\Omega_{2}\right) \mid \eta=0, \quad \frac{\partial w}{\partial n}=0 \text { on } \Gamma \cap \Gamma_{2},\right\} .
$$

Regularity of the domain's boundary $\Gamma_{2}$ (the Lipschitz regularity is sufficient) allows us to state that the estimate

$$
B\left(\Omega_{2}, \eta, \eta\right) \geqslant c_{2}\|\eta\|_{H\left(\Omega_{2}\right)}^{2}, \quad \forall \eta \in H\left(\Omega_{2}\right), \quad \eta=0 \text { on } \Gamma \cap \Gamma_{2},
$$

which is analogous to (7), holds with some constant $c_{2}>0$ independent of $\eta$ [11]. In virtue of (7) and (17), for every fixed $\lambda$ we have

$$
B^{\lambda}\left(\Omega_{\gamma}, \eta, \eta\right) \geqslant c_{\lambda}\|\eta\|_{H\left(\Omega_{\gamma}\right)}^{2} \quad \forall \eta \in H\left(\Omega_{\gamma}\right),
$$


where $c_{\lambda}$ is a constant independent of $\lambda$. It is easy to show that the set $K$ is closed and convex. Besides, one can easily prove that $\Pi_{\lambda}\left(\Omega_{\gamma} ; \eta\right)$ is coercive, convex, weakly lower semicontinuous, and differentiable on $H\left(\Omega_{\gamma}\right)$ [28].

For every fixed $\lambda>0$, by the Weierstrass theorem there exists a solution $\xi^{\lambda}=\left(U^{\lambda}, u^{\lambda}\right)$ to the minimization problem (15). The uniqueness of solutions to (15) follows by the standard arguments [11]. Furthermore, (15) is equivalent to the variational inequality [28]

$$
\xi^{\lambda} \in K, \quad B^{\lambda}\left(\Omega_{\gamma}, \xi^{\lambda}, \eta-\xi^{\lambda}\right) \geqslant \int_{\Omega_{\gamma}} F\left(\eta-\xi^{\lambda}\right) d x \quad \forall \eta \in K
$$

\section{Passage to the limit when a rigidity of the plate goes to infinity.}

Now we are going to make explicit the relationship between the problems (9) and (19). It turns out that the solutions $\xi^{\lambda}$ converge to a limiting function $\tilde{\xi}$ as $\lambda \rightarrow 0$. Moreover, the restriction of $\tilde{\xi}$ onto $\Omega_{1}$ is a solution to the variational inequality (9).

Comparing two inequalities that correspond to (19) with the test functions $\eta=0$ and $\eta=2 \xi$, we get

$$
B\left(\Omega_{1}, \xi^{\lambda}, \xi^{\lambda}\right)+\frac{1}{\lambda} B\left(\Omega_{2}, \xi^{\lambda}, \xi^{\lambda}\right)=\int_{\Omega_{\gamma}} F \xi^{\lambda} d x
$$

From here, making use of $(17),(18)$, for $\lambda \in\left(0, \lambda_{0}\right]$ we have

$$
\left\|\xi^{\lambda}\right\|_{H\left(\Omega_{\gamma}\right)} \leqslant c_{3}, \quad\left\|\xi^{\lambda}\right\|_{H\left(\Omega_{2}\right)} \leqslant c_{4} \lambda
$$

where the constants $c_{3}>0$ and $c_{4}>0$ are independent of $\lambda$. Due to the boundedness of the solutions $\left\{\xi^{\lambda}\right\}, \lambda \in\left(0, \lambda_{0}\right]$ in $H\left(\Omega_{\gamma}\right)$, we can extract a subsequence (we preserve the notation) $\left\{\xi^{\lambda}\right\}$ such that

$$
\xi^{\lambda} \rightarrow \tilde{\xi} \quad \text { weakly in } H\left(\Omega_{\gamma}\right) .
$$

The second estimate in (20) implies

$$
\xi^{\lambda} \rightarrow 0 \quad \text { strongly in } H\left(\Omega_{2}\right)
$$

We show that the restriction $\left.\tilde{\xi}\right|_{\Omega_{1}}$ of $\tilde{\xi}$ onto $\Omega_{1}$ is a solution to the problem (8). We first prove that $\left.\tilde{\xi}\right|_{\Omega_{1}}$ belongs to the set $K_{s}$. The convergence (21) implies the strong convergence of traces

$$
\frac{\partial u^{\lambda}}{\partial \nu} \rightarrow \frac{\partial \tilde{u}}{\partial \nu} \quad \text { strongly in } \quad L_{2}\left(\Gamma_{1} \cap \Gamma_{2}\right), \quad \xi^{\lambda} \rightarrow \tilde{\xi} \quad \text { strongly in } \quad L_{2}\left(\Gamma_{i}\right)^{3}, \quad i=1,2 .
$$

In view of $(22)$, we have $\xi^{\lambda} \rightarrow 0$ in $L_{2}\left(\Gamma_{2}\right)^{3}$. This means that $\tilde{\xi}=0$ in $L_{2}\left(\Gamma_{2}\right)^{3}$. Choosing a subsequence, if necessary, we assume that as $\lambda \rightarrow 0$

$$
\xi^{\lambda} \rightarrow \tilde{\xi}, \quad \frac{\partial u^{\lambda}}{\partial \nu} \rightarrow \frac{\partial \tilde{u}}{\partial \nu} \quad \text { a.e. on } \quad \Gamma_{i}, \quad i=1,2 .
$$

Therefore, we can pass to the limit in the inequalities

$$
\left[U^{\lambda} \nu\right] \geqslant\left[\frac{\partial u^{\lambda}}{\partial \nu}\right], \quad\left[\frac{\partial u^{\lambda+}}{\partial \nu}\right] \geqslant 0 \quad \text { on } \gamma
$$


and, taking into account the equality $\tilde{\xi}=0$ almost everywhere on $\Gamma_{2}$, we obtain the relations

$$
-\tilde{U} \nu \geqslant-\frac{\partial \tilde{u}}{\partial \nu}, \quad \frac{\partial \tilde{u}}{\partial \nu} \leqslant 0 \quad \text { on } \quad \gamma^{-} .
$$

Similarly, we can establish that $\tilde{\xi}=0$ on $\Gamma_{0}$. Thus we have established that the inclusion $\left.\tilde{\xi}\right|_{\Omega_{1}} \in K_{s}$ holds. Choosing the test function $\eta \in K$ such that $\eta=0$ in $\Omega_{2}$, we get from (19)

$$
B\left(\Omega_{1}, \xi^{\lambda}, \eta\right) \geqslant B\left(\Omega_{1}, \xi^{\lambda}, \xi^{\lambda}\right)+\frac{1}{\lambda} B\left(\Omega_{2}, \xi^{\lambda}, \xi^{\lambda}\right)-\int_{\Omega_{2}} F \xi^{\lambda} d x+\int_{\Omega_{1}} F\left(\eta-\xi^{\lambda}\right) d x .
$$

Passing to the lower limit as $\lambda \rightarrow 0$ on both sides of this identity and using (21), (22), we have

$$
B\left(\Omega_{1}, \tilde{\xi}, \eta\right) \geqslant B\left(\Omega_{1}, \tilde{\xi}, \tilde{\xi}\right)+\int_{\Omega_{1}} F(\eta-\tilde{\xi}) d x+\lim _{\lambda \rightarrow 0} \inf \frac{1}{\lambda} B\left(\Omega_{2}, \xi^{\lambda}, \xi^{\lambda}\right) .
$$

The last term in (25) is nonnegative because of (17). This provides to the following inequality

$$
B\left(\Omega_{1}, \tilde{\xi}, \bar{\eta}\right) \geqslant B\left(\Omega_{1}, \tilde{\xi}, \tilde{\xi}\right)+\int_{\Omega_{1}} F(\bar{\eta}-\tilde{\xi}) d x \quad \forall \bar{\eta} \in K_{s} .
$$

It should be pointed out that when deriving the formula (26), we used the fact that by extending an arbitrary function $\bar{\eta} \in K_{s}$ to $\Omega_{2}$ by zero, we obtain the function $\eta \in K$ such that $\eta=0$ in $\Omega_{2}$. We note that (26) coincides up to the notation with (19). By the uniqueness of a solution of the variational inequality (19), we get $\left.\tilde{\xi}\right|_{\Omega_{1}}=\xi_{s}$. The aforesaid remains valid for $\Gamma_{i} \in C^{0,1}, i=1,2$. So, the following statement is proved.

Theorem 3.1 Solutions $\xi^{\lambda}$ of the problems (15) converge weakly in $H\left(\Omega_{\gamma}\right)$ to the function $\tilde{\xi}$ as $\lambda \rightarrow 0$, so that $\left.\tilde{\xi}\right|_{\Omega_{1}}=\xi_{s}$, where $\xi_{\text {s }}$ is the solution of $(8),\left.\tilde{\xi}\right|_{\Omega_{2}}=0$.

\section{Application of the fictitious domains method in a proof of the solvability of an equilibrium problem for a plate with a crack}

This section discusses a problem in which the curve describing the crack, crosses the external boundary at zero angle. In this case, the standard method of proving solvability based on the straightforward application of Korn's inequality could not be applied. This circumstance causes the fact that the boundary contains one boundary cusp, which violates the fulfillment of the Lipschitz condition for a domain of the problem. To study the solvability of such problems, the fictitious domain method, which adapted for this type of problems with one-sided constraints, can be successfully applied.

In order to formulate the problem we start by providing a description of suitable geometric objects. Let $\Omega_{1} \subset R^{2}$ be a bounded connected domain with smooth boundary $\Gamma_{1}$. Suppose that a smooth unclosed curve $\gamma$ lies inside $\Omega_{1}$ such that one of tips of the curve $\bar{\gamma}$ is located on the boundary $\Gamma_{1}$, and an angle between two tangents to curves $\Gamma_{1}, \bar{\gamma}$ at their common point $x^{0}=\left(x_{1}^{0}, x_{2}^{0}\right)$ is zero. The domain with cut is denoted by $\Omega_{1}^{\gamma}=\Omega_{1} \backslash \bar{\gamma}$. In addition, we assume that $\gamma$ can be extended up from the another curve's tip to the outer boundary $\Gamma_{1}$ so that angle between these curves at their point of intersection is positive (see Fig. 4). Denote by $\nu=\left(\nu_{1}, \nu_{2}\right)$ the unit normal vector to $\gamma$. As above, suppose that the plate has a constant thickness equal 


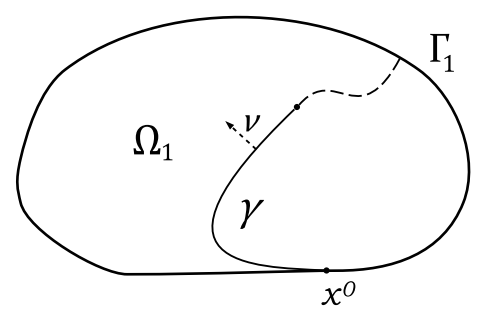

Fig. 4. Geometry of the problem

to 2. Let us assign a three-dimensional Cartesian space $\left\{x_{1}, x_{2}, z\right\}$ with the set $\Omega_{1}^{\gamma} \times[-1,1]$ corresponding to the plate with a crack. The cylindrical surface $x \in \gamma,-1 \leqslant z \leqslant 1$ describes the crack in the plate. As before, the functional of energy for this problem has the following form

$$
\Pi\left(\Omega_{1}^{\gamma}, \chi\right)=\frac{1}{2} B\left(\Omega_{1}^{\gamma}, \chi, \chi\right)-\int_{\Omega_{1}^{\gamma}} F \chi d x, \quad \chi=(W, w),
$$

where the vector $F=\left(f_{1}, f_{2}, f_{3}\right) \in L_{l o c}^{2}\left(\mathbb{R}^{2}\right)^{3}$ is the external force vector [11].

On the exterior boundary $\Gamma_{1}$, we impose the clamping condition

$$
w=\frac{\partial w}{\partial l}=W=0 \text { on } \Gamma_{1}
$$

where $l$ is the unit outward normal to $\Gamma_{1}$. Let us introduce the following notation for the Sobolev spaces:

$$
\begin{gathered}
H^{1,0}\left(\Omega_{1}^{\gamma}\right)=\left\{v \in H^{1}\left(\Omega_{1}^{\gamma}\right) \mid v=0 \text { on } \Gamma_{1}\right\}, \\
H^{2,0}\left(\Omega_{1}^{\gamma}\right)=\left\{v \in H^{2}\left(\Omega_{1}^{\gamma}\right) \mid v=\frac{\partial v}{\partial l}=0 \text { on } \Gamma_{1}\right\}, \\
H\left(\Omega_{1}^{\gamma}\right)=H^{1,0}\left(\Omega_{1}^{\gamma}\right)^{2} \times H^{2,0}\left(\Omega_{1}^{\gamma}\right) .
\end{gathered}
$$

The set of admissible displacements is given by

$$
\hat{K}=\left\{\chi=(W, w) \in H\left(\Omega_{1}^{\gamma}\right) \mid \chi \text { satisfy }(14)\right\} .
$$

It is obvious that the set $\hat{K}$ is convex and closed in $H\left(\Omega_{1}^{\gamma}\right)$. The equilibrium problem for a plate with the nonpenetration condition (14) for a certain known configuration of a plate bending near the crack can be formulated as follows:

$$
\text { find } \hat{\xi} \in \hat{K} \quad \text { such that } \Pi\left(\Omega_{1}^{\gamma} ; \hat{\xi}\right)=\inf _{\eta \in \hat{K}} \Pi\left(\Omega_{1}^{\gamma} ; \eta\right)
$$

The functional $\Pi\left(\Omega_{1}^{\gamma} ; \eta\right)$ is convex and differentiable, hence the problem (27) is equivalent to the variational inequality

$$
\hat{\xi} \in \hat{K}, \quad B_{1}\left(\Omega_{1}^{\gamma}, \hat{\xi}, \chi-\hat{\xi}\right) \geqslant \int_{\Omega_{1}^{\gamma}} F(\chi-\hat{\xi}) d x \quad \forall \chi \in \hat{K}
$$

In order to apply the fictitious domain method, we add to the domain $\Omega_{1}^{\gamma}$ a new domain $\Omega_{2}$ with a smooth boundary $\Gamma_{2}$ so that $x^{0} \in \Sigma, \Sigma=\operatorname{int}\left(\Gamma_{1} \cap \Gamma_{2}\right)$ (see Fig. 5). We denote by $\Omega_{\gamma}=\left(\Omega_{1} \cup \Omega_{2} \cup \Sigma\right) \backslash \bar{\gamma}$, an extended domain with a cut $\gamma$ and by $\Gamma=\left(\Gamma_{1} \cup \Gamma_{2}\right) \backslash \Sigma$ an external boundary of the domain $\Omega_{\gamma}$. Then, we assume that meas $\left(\Gamma \cap \Gamma_{i}\right)>0, i=1,2$. 


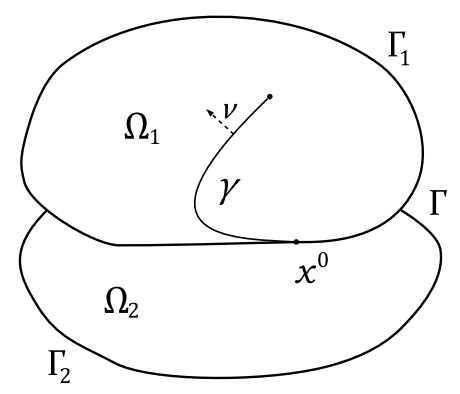

Fig. 5. Geometry of the extended domain

Following the notation adopted in the third section of the article, we denote by $n$ a unit outward normal to $\Gamma$. We formulate the family of auxiliary problems depending on a parameter $\lambda \in\left(0, \lambda_{0}\right]$. For any fixed $\lambda \in\left(0 ; \lambda_{0}\right]$, consider a plate with the mid-plane corresponding to the domain $\Omega_{\gamma}$. Suppose that the elastic coefficients depend on $\lambda$ by formulas (10), moments $m_{i j}^{\lambda}$ and stresses $\sigma_{i j}^{\lambda}, i, j=1,2$, are expressed by the formulas (12). Consider a following energy functional $\Pi_{\lambda}\left(\Omega_{\gamma} ; \eta\right)$ that has the same form as in (13) and defined on $H\left(\Omega_{\gamma}\right)$ ( the space $H\left(\Omega_{\gamma}\right)$ is defined in (11)). So, for any fixed $\lambda \in\left(0 ; \lambda_{0}\right]$, we consider the following variational statement of an equilibrium problem for a plate:

$$
\text { find } \xi^{\lambda} \in K^{\prime} \quad \text { such that } \Pi_{\lambda}\left(\Omega_{\gamma} ; \xi^{\lambda}\right)=\inf _{\eta \in K^{\prime}} \Pi_{\lambda}\left(\Omega_{\gamma} ; \eta\right)
$$

where

$$
K^{\prime}=\left\{\eta \in H\left(\Omega_{\gamma}\right)|\eta=(W, w)| \text { satisfy } \quad(14)\right\}
$$

is the set of admissible functions.

By construction of the domain $\Omega_{\gamma}$, it can be divided into two subdomains $\Omega_{L}^{1}$, $\Omega_{L}^{2}$ with Lipschitz boundaries such that $\gamma \subset \Omega_{L}^{1} \cap \Omega_{L}^{2}$. For each domain $\Omega_{L}^{i}, i=1,2$, we can apply the first Korn and the Poincare inequalities, which give us the following estimates

$$
\begin{gathered}
B\left(\Omega_{2}, \eta, \eta\right) \geqslant c_{2}\|\eta\|_{H\left(\Omega_{2}\right)}^{2} \quad \forall \eta \in H\left(\Omega_{2}\right), \quad \eta=0 \text { on } \Gamma \cap \Gamma_{2}, \\
B^{\lambda}\left(\Omega_{\gamma}, \eta, \eta\right) \geqslant c_{\lambda}\|\eta\|_{H\left(\Omega_{\gamma}\right)}^{2} \quad \forall \eta \in H\left(\Omega_{\gamma}\right),
\end{gathered}
$$

where constants $c_{2}>0, c_{\lambda}>0$ are independent of $\eta$, and the space $H\left(\Omega_{2}\right)$ is defined in the same way as (16).

For any fixed choice of $\lambda \in\left(0 ; \lambda_{0}\right]$, the functional $\Pi_{\lambda}\left(\Omega_{\gamma} ; \eta\right)$ is coercive and weakly lower semicontinuous on $H\left(\Omega_{\gamma}\right)$, and the set $K^{\prime}$ is weakly closed and convex; therefore, the minimization problem (29) has a unique solution $\xi^{\lambda}=\left(U^{\lambda}, u^{\lambda}\right)$ satisfying the variational inequality

$$
\xi^{\lambda} \in K^{\prime}, \quad B^{\lambda}\left(\Omega_{\gamma}, \xi^{\lambda}, \eta-\xi^{\lambda}\right) \geqslant \int_{\Omega_{\gamma}} F\left(\eta-\xi^{\lambda}\right) d x \quad \forall \eta \in K^{\prime} .
$$

A slight modification of the above reasonings of that were carried out section 3 enables us to pass to the limit as $\lambda \rightarrow 0$ in (30) and to prove that $\xi^{\lambda}$ converges to $\xi^{0}$ satisfying $\left.\xi^{0}\right|_{\Omega_{1}}=\hat{\xi}$, where $\hat{\xi}$ is the solution of (28).

Let us show that the problem (28) has a unique solution. To verify the uniqueness we suppose the existence of two solutions $\hat{\xi}_{1} \in K$ and $\hat{\xi}_{2} \in \hat{K}$. Substituting these functions as the test functions into (26), we find 
Adding the last two inequalities, we get

$$
\begin{aligned}
& B\left(\Omega_{1}^{\gamma}, \hat{\xi}_{1}, \hat{\xi}_{2}-\hat{\xi}_{1}\right) \geqslant \int_{\Omega_{1}^{\gamma}} F\left(\hat{\xi}_{2}-\hat{\xi}_{1}\right) d x, \\
& B\left(\Omega_{1}^{\gamma}, \hat{\xi}_{2}, \hat{\xi}_{1}-\hat{\xi}_{2}\right) \geqslant \int_{\Omega_{1}^{\gamma}} F\left(\hat{\xi}_{1}-\hat{\xi}_{2}\right) d x .
\end{aligned}
$$

$$
B\left(\Omega_{1}^{\gamma}, \hat{\xi}_{1}-\hat{\xi}_{2}, \hat{\xi}_{1}-\hat{\xi}_{2}\right)=0
$$

In the following, it will be reasonable to introduce the space $R(O)$ for any domain $O \subset \mathbb{R}^{2}$ :

$$
\begin{gathered}
R(O)=\left\{\zeta(x)=(\rho, \delta) \mid \rho=\left(\rho_{1}, \rho_{2}\right)=\left(-s x_{2}+c_{1}, s x_{1}+c_{2}\right),\right. \\
\left.\delta=a+x_{i} \beta_{i}, \quad x \in O\right\},
\end{gathered}
$$

where reals $a, s, c_{1}, c_{2}, \beta_{1}, \beta_{2} \in \mathbb{R}$ are arbitrary. It is known that the equation

$$
B(O, \eta, \eta)=0
$$

holds if and only if $\eta=\zeta$ in $O$ for some function $\zeta \in R(O)$ (e.g. [4]). Using this property, from (31) we obtain that there exists a function $\zeta \in R\left(\Omega_{1}^{\gamma}\right)$ such that $\hat{\xi}_{1}-\hat{\xi}_{2}=\zeta$ in $\Omega_{1}^{\gamma}$. In view of the relation $\hat{\xi}_{1}-\hat{\xi}_{2}=0$ on $\Gamma_{1}$, we conclude that $\zeta=0$ on $\Gamma_{1}$. Bearing in mind the special structure of $\zeta$, we get $\zeta \equiv 0$ in the whole domain $\Omega_{1}^{\gamma}$. Therefore we have $\hat{\xi}_{1}-\hat{\xi}_{2}=0$ in $\Omega_{1}^{\gamma}$. As a result, the problem (30), which is equivalent to (27) has a unique solution. So, the following statement is proved.

Theorem 4.1 Under the above conditions, there exists a unique solution of the equilibrium problem (27).

The work was supported by Russian Foundation for Basic Research (grant 18-29-10007-mk)

\section{References}

[1] V.D.Stepanov, A.M.Khludnev, The Method of fictitious domains in the signorini problem, Siberian Math. J., 44(2003), no. 6, 1061-1074.

[2] G.V.Alekseev, A.M.Khludnev, Crack in an elastic body crossing the external boundary at zero angle, Vestn. Novosib. Gos. Univ., Ser. Mat. Mekh. Inform. 9(2009), no. 2, 15-29.

[3] K.-H.Hoffmann, A.M.Khludnev, Fictitious domain method for the Signorini problem in a linear elasticity, Adv. Math. Sci. Appl. 14(2004), 465-481.

[4] A.M.Khludnev, Elasticity problems in nonsmooth domains, Moscow, Fizmatlit, 2010 (in Russian).

[5] L.-E.Andersson, A.M.Khludnev, On crack crossing an external boundary. Fictitious domain method and invariant integrals, Sib. J. Industr. Math., 11(2008), no. 3, 15-29.

[6] N.A.Nikolaeva, Method of fictitious areas in a task about balance of a plate of KirchhoffLyava, J. Math. Sci., 221(2017), no. 6, 872-882.

[7] N.P.Lazarev, Fictitious domain method in the equilibrium problem for a Timoshenko-type plate contacting with a rigid obstacle, J. Math. Sci., 203(2014), no. 4, 527-539. 
[8] N.P.Lazarev, H.Itou, N.V.Neustroeva, Fictitious domain method for an equilibrium problem of the Timoshenko-type plate with a crack crossing the external boundary at zero angle Jpn. J. Ind. Appl. Math., 33(2016), no. 1, 63-80.

[9] I.V.Frankina, A contact problem for an elastic plate with a thin rigid inclusion, J. Appl. Industr. Math., 10(2016), no. 3, 333-340.

[10] M.Fabre, J.Pousin, Y.Renard, A fictitious domain method for frictionless contact problems in elasticity using Nitsche's method SMAI J. Comput. Math.,2(2016), 19-50.

[11] A.M.Khludnev, V.A.Kovtunenko, Analysis of cracks in solids, Southampton, WIT-Press, 2000.

[12] N.V.Neustroeva A rigid inclusion in the contact problem for elastic plates, J. Appl. Indust. Math., 4(2010), no. 4, 526-538.

[13] N.P.Lazarev, T.S.Popova, G.A.Rogerson, Optimal control of the radius of a rigid circular inclusion in inhomogeneous two-dimensional bodies with cracks, Z. Angew. Math. Phys. 69(2018), no. 53.

[14] E.M.Rudoy, V.V.Shcherbakov, Domain decomposition method for a membrane with a delaminated thin rigid inclusion, Siberian Electronic Mathematical Reports, 13(2016), no. 1, 395-410.

[15] N.A.Kazarinov, E.M.Rudoy, V.Y.Slesarenko, V.V.Shcherbakov, Mathematical and numerical simulation of equilibrium of an elastic body reinforced by a thin elastic inclusion, Computational Mathematics and Mathematical Physics, 58(2018), no. 5, 761-774.

[16] A.Khludnev, T.Popova, Semirigid inclusions in elastic bodies: Mechanical interplay and optimal control, Computers and Mathematics with Applications, 77(2019), no. 1, 253-262.

[17] A.M.Khludnev, T.S.Popova, On the mechanical interplay between Timoshenko and semirigid inclusions embedded in elastic bodies, Z. Angew. Math. Mech., 97(2017), no. 11, $1406-1417$.

[18] A.M.Khludnev, V.V.Shcherbakov, Singular path-independent energy integrals for elastic bodies with Euler-Bernoulli inclusions, Math. Mech. Solids., 22(2017), no. 11, 2180-2195.

[19] A.M.Khludnev, V.V.Shcherbakov, A note on crack propagation paths inside elastic bodies, Applied Mathematics Letters, 79(2018), no. 1, 80-84.

[20] N.P.Lazarev, E.M.Rudoy, Optimal size of a rigid thin stiffener reinforcing an elastic plate on the outer edge, Z. Angew. Math. Mech., 97 (2017), no. 9, 1120-1127.

[21] E.M.Rudoy, Domain decomposition method for a model crack problem with a possible contact of crack edges, Computational Mathematics and Mathematical Physics, 55(2015), no. 2, 305-316.

[22] E.V.Pyatkina, On control problem for two-layers elastic body with a crack, J. Math. Sci., 230(2018), no. 1, 159-166. 
[23] A.I.Furtsev, Differentiation of energy functional with respect to delamination's length in problem of contact between plate and beam, Siberian Electronic Mathematical Reports 15(2018), 935-949.

[24] H.Itou, V.A.Kovtunenko, K.R.Rajagopal, Well-posedness of the problem of non-penetrating cracks in elastic bodies whose material moduli depend on the mean normal stress, International Journal of Engineering Science 136(2019), 17-25.

[25] H.Itou, V.A.Kovtunenko, K.R.Rajagopal, Nonlinear elasticity with limiting small strain for cracks subject to non-penetration, Mathematics and Mechanics of Solids, 22(2017), no. 6, $1334-1346$.

[26] P.N.Vabishchevich, The Method of Fictitious Domains in Problems of Mathematical Physics, Moscow, Moscow. State Univ. Press, 1991 (in Russian).

[27] J.N.Reddy, Theory and analysis of elastic plates and shells, 2nd edn. Boca Raton, CRC Press/Taylor and Francis, 2007.

[28] C.Baiocchi, A.Capelo, Variational and quasivariational inequalities. Applications to free boundary problems. Chichester, Wiley, 1984.

\title{
Метод фиктивных областей в задаче о равновесии пластины Кирхгофа-Лява с условиями непроникания для известной конфигурации изгиба
}

\author{
Нюргун П. Лазарев \\ Владимир В. Эверстов \\ Наталья А. Романова \\ Институт математики и информатики \\ Северо-Восточный федеральный университет \\ Белинского, 58, Якутск, 677000 \\ Россия
}

$\bar{B}$ работе исследуются новые модели о равновесии пластин с условиями непроникания типа Синъорини. Предполагается, что под действием специальных нагрузок пластины имеют деформации с определенной заранее известной конфигурацией кромок. Для этого частного случая мы рассматриваем новые условия непроникания, которые позволяют нам более точно описать контактное взаимодействие на кромках. С помощью метода фиктивных областей доказано, что исходную контактную задачу можно получить с помощъю предельного перехода по параметру жесткости в семействе вспомогательных задач, сформулированных в более широкой области. Задачи семейства моделируют равновесие пластины с трещиной и зависят от положительного параметра жесткости. При этом на внутренней границе, соответствующей трещине, налагаются условия непроникания противоположных берегов трещины в виде неравенств. Для задачи о пластине с трещиной, выходящей под нулевым углом на внешнюю граничу (случай границы с одним каспом), доказана ее однозначная разрешимость.

Ключевые слова: краевые условия Синворини, фиктивная область, условия непроникания, пластина Кирхгофа-Лява, трещина. 